\title{
CONSERVED QUANTITIES FOR AXISYMMETRIC CAVITIES NEAR BOUNDARIES
}

\author{
R. PAUll and J.R. Blake
}

In axisymmetric irrotational flows of a perfect fluid under gravity there are three basic conserved quantities; axial momentum, energy and a circulation based, radial moment of momentum. This paper adapts these conservation principles to describe cavity collapse adjacent to a rigid boundary in a semi-infinite perfect fluid. They afford a global model accounting for volume change, migration and jet formation; physically the most significant features of bubble collapse close to a rigid boundary.

\section{INTRODUCTION}

In recent papers Longuet-Higgins [7, 8], Benjamin [2] and Benjamin and Olver [3] have presented conserved scalar quantities applicable to perfect, irrotational flows under gravity in two and three dimensions. In axisymmetric flow these principles concern conservation of mass, mean axial position, axial momentum (Kelvin impulse), energy and a circulation based, radial moment of momentum (virial). Although the latter is relatively unfamiliar, its existence is of importance when developing global models since it provides a further clamp on flow characteristics. This is nowhere more apparent than in the problem of cavity collapse adjacent to a rigid boundary, as this paper aims to demonstrate.

A cavity, under constant internal pressure, collapsing adjacent to an infinite plane boundary in an otherwise semi-infinite irrotational perfect fluid is considered. Implementation of the conservation principles in this problem relies on generalising their definitions to integral expressions over the bubble surface. As a result of the boundary conditions, the time rates of change of the conserved quantities appear as simple surface integrals involving the velocity and velocity potential. These identities incorporate the constant internal cavity pressure and the boundary condition of the plane surface. Cavity geometries during collapse, however, are known to exhibit an inner jet region and an outer, almost spherical, Rayleigh region. A minimum global description would thus hope to determine radius and centre of the outer region, jet width, length and fullness. The five conservation principles would thus provide just sufficient conditions

Received 28 March 1989

This research has been supported by the Australian Research Grants Scheme

Copyright Clearance Centre, Inc. Serial-fee code: 0004-9729/90 \$A2.00+0.00. 
to determine a simple global model for cavitation. A more detailed discussion on the conservation of linear momentum (the Kelvin impulse) may also be found in the review paper by Blake [4], while an approximate method to model the growth and collapse of cavitation bubbles near a rigid boundary have recently been developed by Kucera and Blake [6].

\section{Conservation quantities}

The geometry of the problem considered appears in cross-section as in Figure 1.

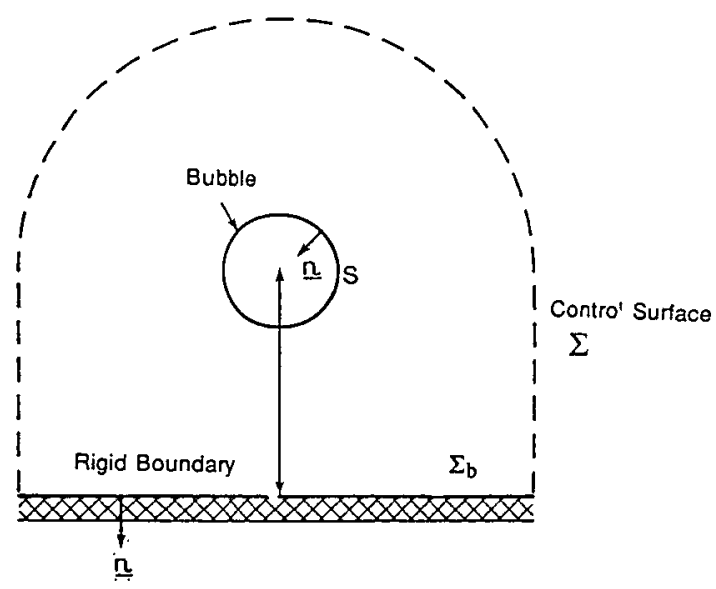

Figure 1. Geometry used in theory

Here the surface of the cavity is $S$ with $(x, r)$ being cylindrical polar coordinates. The axis of symmetry aligns with the $x$ axis and is perpendicular to the boundary $\Sigma_{b}$. The control surface in the fluid will be denoted by $\Sigma$ and all surface normals $\mathbf{n}$ will be directed out of the fluid.

Only perfect irrotational flows in a uniform gravitational field aligned with the axis of symmetry are to be considered. The equations governing such flows in terms of the velocity potential $\phi$ are

$$
\frac{\partial \phi}{\partial t}+\frac{p}{\rho}+\frac{1}{2}|\mathbf{u}|^{2}+g x=\frac{p_{\infty}}{\rho}
$$

and

$$
\nabla^{2} \phi=0
$$


where $p$ is the pressure, $\rho$ is the fluid density, $g$ is the gravitational acceleration in the negative $x$ direction and

$$
\mathbf{u}=\nabla \phi
$$

is the velocity. In (2.1) the velocity potential has been normalised so as to vanish at infinity in line with the absence of an external convection field;

$$
\phi=0\left(\frac{1}{R}\right) \quad \text { as } \quad R \rightarrow \infty\left(R^{2}=x^{2}+r^{2}\right) .
$$

The remaining boundary conditions are that

$$
p=p_{c} \quad(\text { a constant })
$$

on $S$, the bubble surface. The cavity pressure $p_{c}$ is normally equated to the saturated vapour pressure for 'cold' liquids. Other equations of state may be used for the gas or vapour phase depending on physical circumstances. There is no flow through the rigid plane boundary $\Sigma_{b}$,

$$
\mathbf{u} \cdot \mathbf{n}=\mathbf{0} \text { on } \boldsymbol{x}=\mathbf{0} .
$$

The work of Benjamin and Olver [2] now indicates that in general three dimensional flow the complete set of conserved quantities consists of mass, mean position (3 components), momentum ( 3 components), angular moment of momentum ( 3 components), circulation based radial moment of momentum ( 1 component) and energy. The condition of axisymmetry applicable here reduces several of these to trivial statements. In terms of a closed material surface $S^{*}$ (outward normal $\mathbf{n}$ ) surrounding a volume $V$ of fluid consisting of the inner boundary $S$ of the cavity and an exterior boundary $\Sigma_{b} U \Sigma$, the following integral formulations provide useful information: Mass $M^{*}$,

$$
M^{*}=\rho \int_{V} d V=\rho \oint_{S^{*}} \frac{1}{3} \mathbf{x} \cdot \mathbf{n} d S
$$

Mean Axial Position $\bar{x}^{*}$,

$$
M \bar{x}^{*}=\rho \int_{V} x d V=\rho \oint_{S^{*}} \frac{1}{2} \mathrm{x}^{2} \mathrm{e}_{x} \cdot \mathbf{n} d S
$$

Axial Momentum $I^{*}$,

$$
I^{*}=\rho \int_{V} \mathbf{u} \cdot \mathbf{e}_{x} d V=\rho \oint_{S^{*}} \phi\left(\mathbf{e}_{x} \cdot \mathbf{n}\right) d S
$$


Circulation based, radial moment of momentum $B^{*}$,

$$
B^{*}=\rho \int_{V}\left(\frac{1}{3} \mathbf{x} \cdot \mathbf{u}+\phi\right) d V=\rho \oint_{S^{*}} \phi \frac{1}{3} \mathbf{x} \cdot \mathbf{n} d S
$$

Energy E*,

$$
E^{*}=\rho \int_{V}\left(\frac{1}{2} \mathbf{u}^{2}+g x\right) d V=\rho \oint_{S^{*}}\left[\frac{1}{4} \nabla \phi^{2}+\frac{g}{2} x^{2} \mathbf{e}_{x}\right] \cdot \mathbf{n} d S .
$$

Here $\mathbf{x}$ is the position vector of a point relative to a chosen origin. The relations between the volume and surface integrals are easily obtained from the divergence theorem.

In the problem to be considered here it is not possible to apply the conservation principles of Benjamin and Olver [3] directly to a semi-infinite fluid since divergent integrals for most of the above quantities result as a consequence of $\phi=0(1 / R)$ as $R \rightarrow \infty$. It should be noted, however, that each of the conserved quantities possesses a surface integral representation which allows them to be defined for any material surface surrounding a finite volume $V$. Since the surface of the cavity $S$ is one of material surfaces enclosing the fluid volume $V$, we may instead define the 'conserved quantities' to be over $S$ alone and thence relate the integrals to the outer boundaries by using (2.7) to (2.11). This will provide specialised information about the cavity dynamics.

\section{Material surface integral PROPERTIES}

Often properties based on an integral over the surface of the bubble are physically meaningful quantities (for example, bubble centroid, Kelvin impulse). Thus in this section we consider integrals over the surface of the bubble which may be exploited further by taking advantage of the conservation quantities discussed in the previous section.

The conservation principles of Benjamin and Olver [3] alter slightly with these new definitions since, for example, the cavity volume is no longer constant and for quantities to be positive a sign change may be necessary. To derive the new conservation results expressions are required for the time rate of change of the material surface integrals (2.7) to (2.11). The transport identity for an incompressible fluid,

$$
\begin{aligned}
\frac{d}{d t}(\mathbf{n} d S) & =\mathbf{n} d S \cdot\left[(\nabla \cdot \mathbf{u}) \mathbf{I}-(\nabla \mathbf{u})^{T}\right] \\
& =-\mathbf{n} d S \cdot \nabla \nabla \phi
\end{aligned}
$$

is useful in this regard (see also Batchelor [1]). After some manipulation it follows that

$$
\begin{aligned}
\frac{d M_{c}}{d t} & =-\rho \int_{S} \frac{1}{3} \mathbf{n} d S \cdot[3 \mathbf{u}+\nabla \times(\mathbf{x} \times \mathbf{u})] \\
& =-\rho \int_{S} \mathbf{u} \cdot \mathbf{n} d S
\end{aligned}
$$


(that is, the rate of change of displaced mass in the bubble $V$ is equal to the mass flow across the bubble surface $S$ ). From the boundary condition in (2.6), it also follows that,

$$
\frac{d M_{c}}{d t}=\rho \int_{\Sigma} \mathbf{u} \cdot \mathbf{n} d S
$$

leading to the obvious statement of incompressibility,

$$
\frac{d M^{*}}{d t}=0
$$

The rate of change of the 'centre of mass' leads to the following relations,

$$
\begin{aligned}
\frac{d\left(M_{c} \overline{\mathbf{x}}\right)}{d t} & =-\rho \int_{S} \mathbf{n} d S \cdot\left[\mathbf{u} x+\nabla \times\left(\frac{1}{2} \mathbf{x}^{2} \mathbf{e}_{x} \times \mathbf{u}\right)\right], \\
& =-\rho \int_{S} x \mathbf{u} \cdot \mathbf{n} d S,
\end{aligned}
$$

The rate of change of axial momentum (Kelvin Impulse) yields ([5])

$$
\frac{d I}{d t}=\rho \int_{S}\left[\frac{d \phi}{d t} \mathbf{e}_{x}-\phi \mathbf{e}_{x} \cdot \nabla \nabla \phi\right] \cdot \mathbf{n d S} .
$$

Likewise the rate of change of the circulation based radial moment of momentum yields

$$
\frac{d B}{d t}=\frac{\rho}{3} \int_{S}\left[\frac{d \phi}{d t} \mathbf{x}+\phi \nabla \phi-\phi \mathbf{x} \cdot \nabla \nabla \phi\right] \cdot \mathbf{n} d S .
$$

If $\mathrm{E}$ is first decomposed as

$$
E=T+M_{\mathrm{c}} g \bar{x}
$$

where the expression for the kinetic energy of the fluid reduces to a surface integral over the bubble, because the integrals over both $\sum_{b}$ and $\Sigma$ are zero,

$$
T=\rho \int_{S} \frac{1}{2} \phi \nabla \phi \cdot \mathbf{n} d S=\int_{V} \frac{1}{2} \rho \mathbf{u}^{2} d V
$$

then $d E / d t$ is given by the sum of the multiple of $g$ and (3.6) and

$$
\frac{d T}{d t}=\rho \int_{V}\left[\frac{d}{d t}\left(\frac{1}{2} \mathbf{u}^{2}\right)+\frac{1}{2} \mathbf{u}^{2} \nabla \cdot \mathbf{u}\right] d V .
$$


In (3.3) to (3.11) suppression of solenoidal terms follows from single valuedness of operands over the cavity surface. As a consequence of cavity contractions, it should be noted that

$$
\frac{d M_{c}}{d t} \neq 0 \text { and } \frac{d}{d t} M_{c} \bar{x} \neq I,
$$

which contrast with equalities in Benjamin and Olver [3]. The two conservation principles (3.3) and (3.4) provide kinematic conditions on the cavity collapse. The dynamics of collapse are included by manipulating further (3.5) using (2.1), (2.2) and the boundary conditions.

For axial momentum, it follows from (3.7) that

$$
\frac{d I}{d t}=\rho \int_{S}\left[\frac{1}{2}(\nabla \phi)^{2} \mathbf{e}_{x}-\phi \mathbf{e}_{x} \cdot \nabla \nabla \phi-g x \mathbf{e}_{x}\right] \cdot \mathbf{n} d S
$$

as a consequence of the definition of the substantial derivative, the Bernoulli pressure condition and a constant pressure difference between cavity and infinity. In line with Blake and Cerone [5], the identity

$$
\nabla \times(\phi \nabla \phi \times \mathbf{I})=\phi \nabla \nabla \phi+\nabla \phi \nabla \phi-\left[(\nabla \phi)^{2}+\phi \nabla^{2} \phi\right] \mathbf{I}
$$

then yields, in view of $(2.2)$, that

$$
\frac{d I}{d t}=\rho \int_{S} \mathbf{e}_{x} \cdot\left(\mathrm{uu}-\frac{1}{2} \mathrm{u}^{2} \mathrm{I}\right) \cdot \mathrm{n} d S+g M,
$$

provided the potential $\phi$ is a single valued function over the cavity surface. (If the cavity were to become multi-connected, (3.12) would require modification.) The surface integral in (3.15) may be replaced by an integral over the plane boundary yielding [5],

$$
\frac{d I}{d t}=-\rho \int_{\sum_{b}} \mathbf{e x} \cdot\left(\mathrm{uu}-\frac{1}{2} \mathrm{u}^{2} \mathrm{I}\right) \cdot \mathrm{n} d S+g M=-\pi \rho \int_{0}^{\infty} r v^{2} d r+g M,
$$

where $v$ is the velocity parallel to the plane boundary. This results from the identity

$$
\nabla \cdot\left(\mathrm{uu}-\frac{1}{2} \mathrm{u}^{2} I\right)=(\nabla \cdot \mathrm{u}) \mathrm{u}+(\nabla \times \mathrm{u}) \times \mathrm{u}=0
$$

within the fluid, leading to the change in sign in (3.15)..

The dynamics of the motion (2.1) allow (3.8) to be rewritten similarly

$$
\frac{d B}{d t}=\left(\frac{p_{\infty}-p_{c}}{\rho}\right) M-\frac{4}{3} M g \bar{x}+\frac{\rho}{3} \int_{S}\left[\left(\frac{1}{2}(\nabla \phi)^{2} \mathbf{I}-\phi \nabla \nabla \phi\right) \cdot \mathbf{x}+\phi \nabla \phi\right] \cdot \mathbf{n} d S .
$$


The identity (3.16) then indicates

$$
\begin{aligned}
\frac{d B}{d t} & =\left(\frac{p_{\infty}-p_{c}}{\rho}\right) M_{c}-\frac{4}{3} M g \bar{x}-2 T+\frac{\rho}{3} \int_{S} \mathbf{x} \cdot\left(\mathrm{uu}-\frac{1}{2} \mathbf{u}^{2} I\right) \cdot \mathrm{n} d S \\
& =\left(\frac{p_{\infty}-p_{c}}{\rho}\right) M_{c}-3 M_{c} g \bar{x}+\frac{5}{3} E
\end{aligned}
$$

If we note that $M_{c}=\rho V_{c}$, where $V_{c}$ is the volume of the cavity then (3.17) becomes

$$
\frac{d B}{d t}=\left(p_{\infty}-p_{c}\right) V_{c}-3 \rho g \bar{x} V_{c}+\frac{5}{3} E,
$$

a type of energy integral. A result similar to this has recently been derived by [8],

Equation (2.1) also indicates that, from (3.11),

$$
\begin{aligned}
\frac{d T}{d t} & =-\int_{V} \nabla \cdot[\mathbf{u}(p+\rho g x)] d V \\
& =-\int_{S} \mathbf{n} d S \cdot \mathbf{u}(p+\rho g x)-\int_{\Sigma} \mathbf{n} d S \cdot \mathbf{u}(p+\rho g x),
\end{aligned}
$$

since $u \cdot n=0$ on the plane boundary. On the surface $\sum$ we have the following,

$$
p+\rho g x=p_{\infty}-\frac{\partial \phi}{\partial t}-\frac{1}{2} \mathbf{u}^{2}=p_{\infty}+O\left(\frac{1}{R}\right) \quad \text { and } \quad \mathbf{u}=O\left(\frac{1}{R^{2}}\right)
$$

so that the only contribution from the integral over $\sum$ in (3.19) comes from the $p_{\infty}$ term. Thus (3.19) becomes

$$
\frac{d T}{d t}=\frac{\left(p_{c}-p_{\infty}\right)}{\rho} \frac{d M}{d t}+g \frac{d}{d t}(M \bar{x})
$$

whence

$$
\frac{d E}{d t}=\left(\frac{p_{c}-p_{\infty}}{\rho}\right) \frac{d M}{d t}
$$

On integration this leads to the well known expression for the energy of,

$$
E_{0}=E+\left(p_{\infty}-p_{c}\right) V
$$

where $E_{0}$ is constant throughout the lifetime of the bubble. 


\section{Conclusions}

These global conservation quantities are of considerable value in modelling cavitation phenomena. Firstly they may be used as global checks on large scale numerical calculations on bubbles near rigid boundaries. As an alternative they may be exploited in developing approximate cavitation bubble models instead of using much larger numerical simulation programs based on the boundary integral method. In addition these global quantities give a valuable insight into the response of cavitation bubbles near boundaries. In the case of the Kelvin impulse this may be used to predict the direction of migration [4].

\section{REFERENCES}

[1] G.K. Batchelor, An introduction to fluid dynamics (Cambridge University Press, Cambridge, 1967).

[2] T.B. Benjamin, 'Hamiltonian theory for motions of bubbles in an infinite liquid', J. Fluid Mech. 181 (1987), 349-379.

[3] T.B. Benjamin and P.J. Olver, 'Hamiltonian structure, symmetries and conservation laws for water waves', J. Fluid Mech. 125 (1983), 137-185.

[4] J.R. Blake, 'The Kelvin impulse: application to cavitation bubble dynamics', J. Austral. Math. Soc. (Ser. B) 30 (1988), 127-146.

[5] J.R. Blake and P. Cerone, 'A note on the impulse due to a vapour bubble near a boundary', J. Austral. Math. Soc. (Ser. B) 23 (1982), 383-393.

[6] A. Kucera and J.R. Blake, 'Approximate methods for modelling the growth and collapse of cavitation bubbles near boundaries', Bull. Austral. Math. Soc. 41 (1990), 1-44.

[7] M.S. Longuet-Higgins, 'On integrals and invariants for inviscid, irrotational flow under gravity', J. Fluid Mech. 134 (1983), 155-159.

[8] M.S. Longuet-Higgins, 'Some integral theorems relating to the oscillation of bubbles', $J$. Fluid Mech. 204 (1989), 159-166.

12 Marshall Street

Kingston Qld 4114

Australia

\author{
Department of Mathematics \\ The University of Wollongong \\ Wollongong NSW 2500 \\ Australia \\ Current address: \\ School of Mathematics and Statistics \\ The University of Birmingham \\ Edgbaston B15 2TT \\ United Kingdom
}

